\title{
Elucidation of the Mechanisms and Molecular Targets of Sanhuang Xiexin Decoction for Type 2 Diabetes Mellitus Based on Network Pharmacology
}

\author{
Manman Xu $\mathbb{D}^{1},{ }^{1}$ Zhonghao Li $\mathbb{D},{ }^{2}$ Lu Yang $\mathbb{D}^{3},{ }^{3}$ Wujianwen Zhai ${ }^{(D)},{ }^{1}$ Nina Wei $\left(\mathbb{D},{ }^{3}\right.$ \\ Qiuyan Zhang $\mathbb{D}^{1}{ }^{1}$ Bin Chao, ${ }^{1}$ Shijing Huang $\mathbb{D}^{1},{ }^{1}$ and Hanming Cui $\mathbb{D}^{1}$ \\ ${ }^{1}$ Research and Development Center of Traditional Chinese Medicine, Guangan'men Hospital, China Academy of Chinese \\ Medical Sciences, Beijing 100053, China \\ ${ }^{2}$ Department of Neurology, Dongfang Hosipital Beijing University of Chinese Medicine, Beijing 100078, China \\ ${ }^{3}$ Shaanxi University of Chinese Medicine, Department of Traditional Chinese Medicine, First Clinical Medical College, \\ 712000 Shaanxi, China
}

Correspondence should be addressed to Shijing Huang; gamhsj@126.com and Hanming Cui; cui-yaoshi@163.com

Received 11 March 2020; Accepted 10 June 2020; Published 10 August 2020

Academic Editor: Yasuyuki Kihara

Copyright (C) 2020 Manman Xu et al. This is an open access article distributed under the Creative Commons Attribution License, which permits unrestricted use, distribution, and reproduction in any medium, provided the original work is properly cited.

Sanhuang Xiexin Decoction (SXD) is commonly used to treat type 2 diabetes mellitus (T2DM) in clinical practice of traditional Chinese medicine (TCM). In order to elucidate the specific analysis mechanisms of SXD for T2DM, the method of network pharmacology was applied to this article. First, the effective ingredients of SXD were obtained and their targets were identified based on the TCMSP database. The T2DM-related targets screened from the GEO database were also collected by comparing the differential expressed genes between T2DM patients and healthy individuals. Then, the common targets in SXD-treated T2DM were obtained by intersecting the putative targets of SXD and the differential expressed genes of T2DM. And the protein-protein interaction (PPI) network was established using the above common targets to screen key genes through protein interactions. Meanwhile, these common targets were used for GO and KEGG analyses to further elucidate how they exert antidiabetic effects. Finally, a gene pathway network was established to capture the core one in common targets enriched in the major pathways to further illustrate the role of specific genes. Based on the data obtained, a total of 67 active compounds and 906 targets of SXD were identified. Four thousand one hundred and seventy-six differentially expressed genes with a $P$ value $<0.005$ and $\mid \log 2$ (fold change) $\mid>0.5$ were determined between T2DM patients and control groups. After further screening, thirty-seven common targets related to T2DM in SXD were finally identified. Through protein interactions, the top 5 genes (YWHAZ, HNRNPA1, HSPA8, HSP90AA1, and HSPA5) were identified. It was found that the functional annotations of target genes were associated with oxygen levels, protein kinase regulator, mitochondria, and so on. The top 20 pathways including the PI3K-Akt signaling pathway, cancers, HIF-1 signaling pathway, and JAK-STAT signaling pathway were significantly enriched. CDKN1A was shown to be the core gene in the gene-pathway network, and other several genes such as CCND1, ERBB2, RAF1, EGF, and VEGFA were the key genes for SXD against T2DM. Based on the network pharmacology approach, we identified key genes and pathways related to the prognosis and pathogenesis of T2DM and also provided a feasible method for further studying the chemical basis and pharmacology of SXD.

\section{Introduction}

Type 2 diabetes mellitus (T2DM) is currently one of the most important noncommunicable diseases that threaten human health in the current medical field due to its rapidly increas- ing morbidity and mortality. According to epidemiological surveys, the number of T2DM patients worldwide reached 415 million in 2015, of which $75 \%$ were in the developing countries. It is estimated that by 2040 , the world will have nearly 642 million people with diabetes [1].With the growing 
research level of the pathological mechanisms of T2DM, plenty of pharmacological interventions targeting its mechanism of action have been widely used in the clinic to delay its progression [2]. The clinical characteristics of T2DM are mainly chronic glucose metabolism disorders. Its chronic hyperglycemia can cause long-term damage to various organs, which seriously affects the quality of life. However, most drugs for treating T2DM have certain adverse reactions, such as lactic acidosis, hypoglycemia, liver damage, and allergies, [3] which tend to be more evident with the time of treatment. In addition, the medical costs of T2DM and its complications also place a heavy financial burden on individuals, families, and governments. Thus, it has been the global research focus to search for more effective and safe drugs for the treatment of T2DM.

In recent years, with the continuous deepening of understanding and research on T2DM, traditional Chinese medicines (TCMs) have played a unique advantage in treating T2DM and its chronic complications, such as Guizhi Shaoyao Zhimu decoction [4], Liuwei Dihuang Pill [5], and Shenqi Dihuang decoction [6]. Sanhuang Xiexin decoction (SXD) is a classic prescription of three traditional herbal extracts including Rheum palmatum L. (Dahuang), Scutellaria baicalensis Georgi (Huangqin), and Coptis chinensis Franch. (Huanglian) at a ratio of $2: 1: 1$, which has been used to cure diabetes since the Tang Dynasty (sixth century C.E.) [7]. In some clinical trials, SXD can not only significantly reduce fasting blood glucose and basal insulin dose [8] but also reduce weight and improve T2DM symptoms [9]. In addition, another clinical research conducted by $\mathrm{Zhu}$ et al. [10] found that the effective rates of improving insulin peripheral resistance and reducing blood sugar in the SXD group were $79.2 \%$ and $80.1 \%$, mainly by means of reducing hyperinsulinemia and improving insulin sensitivity. Many research have reported that the major constituents of SXD includes berberine, palmatine, baicalin, baicalein, and coptisine, which can effectively exert bioactivities including antiinflammation [11], gastric protection [12], antioxidation [13], antiatherosclerosis [14], and serum cholesterol reduction and benefits to early-stage diabetic nephropathy [15]. However, the possible molecular mechanism of the effective active ingredients in SXD against T2DM has been rarely studied, and the specific targets of exerting pharmacological effects are unknown, which becomes the main restriction on its wider application.

The TCM formula is usually composed of multiple drugs, so they have the characteristics of multicomponent, multipathway, and multitarget synergy [16]. However, it also brings the disadvantages of the specific single herb with unclear efficacy and unclear mechanisms of action due to this complexity, making it difficult for us to conduct a comprehensive and systematic study from the whole to the level of tissues, organs, cells, and molecules. With the rapid development of computational science, genomics, proteomics, and metabolomics, the concept of network pharmacology was presented to the public as a new interdisciplinary science, [17] which can reveal the effect mechanism of multimolecular drugs synergistically acting on the human body. In the present study, the network pharmacology approaches were used to elucidate the mechanisms and molecular targets of SXD for T2DM based on some data resources and tools.

\section{Materials and Methods}

2.1. Active Ingredient Screening. First, we used the Traditional Chinese Medicine Systems Pharmacology Database and Analysis Platform (TCMSP: https://sm.nwsuaf.edu.cn/lsp/ tcmsp.php.) to screen effective compounds in SXD. At the same time, we use absorption, distribution, metabolism, and excretion (ADME) to select active ingredients with therapeutic effects, so we set the indicators as oral bioavailability $(\mathrm{OB}) \geq 30 \%$ [18] and drug-likeness (DL) $\geq 0.18$ [19]. TCMSP is currently one of the largest Chinese medicine databases, which consists of all the 499 Chinese herbs registered in the Chinese Pharmacopoeia with 29384 ingredients, 3311 targets, and 837 associated diseases [20]. And it is also a unique Chinese herbal medicine system pharmacology database which can acquire the herbs, chemicals, targets, and drug-target networks. Eventually, sixty- seven eligible compounds were obtained, 16 in Dahuang, 14 in Huanglian, and 37 in Huangqin.

2.2. Identify the Targets of the Active Ingredients. The DrugBank database [21] (https://www.drugbank.ca/) was used to identify the targets of the active ingredients in SXD. Two thousand four hundred and fifty-three targets were obtained from the database, 654 in Dahuang, 596 in Huanglian, and 1203 in Huangqin. Then, we continued to collect distinct targets related to the active ingredients in SXD after discarding duplicate data. Eventually, a total of 906 targets were collected after intersecting with the above 67 active ingredients, including 110 in Dahuang, 287 in Huanglian, and 509 in Huangqin.

2.3. Identify Targets for T2DM. Known differential expressed targets related to T2DM were acquired from the GEO database (https://www.ncbi.nlm.nih.gov/geo/, Series: GSE29221, Platform: GPL6947, 24 Samples), using "type 2 diabetes mellitus" as the keyword. Then, the genes with a $P$ value < 0.05 and $\mid \log 2$ (fold change) $\mid>0.5$ were considered as target genes with significantly differential expression in T2DM patients.

2.4. Compound-Target Network Was Established. The common targets in SXD-treated T2DM were obtained by intersecting the putative targets of active compounds in SXD and the differential expressed genes of T2DM.To visualize the relationship between active compounds in SXD and the common targets, a compound-target network was built to reflect the graphical interactions using Cytoscape software [22] (version 3.7.1, Boston, MA, USA). The compoundtarget network consists of nodes and edges, in which nodes represent molecules that mainly refer to the compounds in SXD and the common targets, and edges indicate intermolecular interactions that mainly refer to the connections between compounds and targets.

2.5. PPI Network Was Established. The protein-protein interaction (PPI) network was constructed with the above 
common targets using the plugin Bisogenet [23] of Cytoscape 3.5.1 software. And the PPI data in the network came from the following databases: Biological General Repository for Interaction Datasets (GRID), Biological General Repository for Interaction Datasets (BioGRID), Human Protein Reference Database (HPRD), Biomolecular Interaction Network Database (BIND), Molecular Interaction Database (MINT), and Database of Interacting Proteins (DIP). To further screen out key targets that play an important role in the pathogenesis of T2DM, the nodes with topological importance in the PPI network were filtered through a series of parameters using Cytoscape plugin CytoNCA, such as degree centrality (DC), betweenness centrality (BC), closeness centrality (CC), eigenvector centrality (EC), local average connectivity-based method (LAC), and network centrality (NC). After a series of screening, we finally got a PPI network containing core genes, and on the other hand, it also showed the interaction between the important proteins.

2.6. GO and KEGG Analyses. Annotation, Visualization and Integrated Discovery database (DAVID, https://david .ncifcrf.gov, v6.8) [24] was used for GO analysis with biological processes, cellular components, and molecular functions. Among them, the functional categories with enriched genes $($ FDR $<0.05)$ were screened and then we select the top 20 GO functional categories for analysis. Similarly, the pathways with enriched genes (FDR < 0.05) were confirmed for further analysis using DAVID which assigned the Kyoto Encyclopedia of Genes and Genomes (KEGG) database.

2.7. Gene-Pathway Network Was Established. Based on the results of KEGG analysis, the enriched genes in the top 20 pathways were further selected for gene-pathway network analysis using Cytoscape software. This network can intuitively show the relationship between the genes and pathways, and screen for key genes that play an important role in the process of T2DM. Finally, the gene-pathway network was constructed, in which the size of nodes represents the topological importance of genes and pathways.

\section{Results}

3.1. Putative Targets of SXD and T2DM Analysis. Through the screening of the TCMSP database, 67 SXD compounds which meet the requirements were finally selected as the candidate compounds (Table 1). From the GEO database, 25136 T2DM-related targets were acquired, which proved to be involved in the pathological process of T2DM. According to the obtained differential expression genes, a volcano map was drawn to better show the distribution of genes, in which there were red upregulated and green downregulated dots. As shown in Figure 1, we obtained a total of 4176 differential expressed genes, including 2271 downregulated genes and 1905 upregulated genes.

3.2. Compound-Target Network Analysis. Through the intersection between the putative targets of active compounds in SXD and the differential expressed genes of T2DM, 37 common targets were identified. Through screening and removal of duplicates, there are 25 compounds related to T2DM in
SXD, of which there are 3 compounds in Dahuang, 4 in Huanglian, 17 in Huangqin, and a common compound from Dahuang and Huangqin. As shown in Figure 2, the compound-target network was established with the 37 common targets using Cytoscape software. The network contained 62 nodes $(25$ compounds in SXD and 37 common targets) and 66 edges which indicated the compound-target interactions. According to the degree, the compounds with the largest three ranking values in SXD were MOL000098 quercetin in Huanglian, MOL000449 aloe-emodin in Dahuang, and MOL000471 stigmasterol in Huangqin, respectively. In addition, the $\mathrm{OB}$ of quercetin, aloe-emodin, and stigmasterol is $46.43,83.38$, and $43.83 \%$, respectively. Therefore, the three compounds may be key active ingredients of SXD due to their important position in the compound-target network.

3.3. PPI Network Analysis. The PPI network is a relational network of biomolecules, and it plays an important role in the biological processes. Therefore, if the functional modules in the PPI network were identified, it could help understand the mechanism of biological activity and the pathogenesis of diseases [25]. Therefore, a PPI network of the common targets was constructed using PPI data. As shown in Figure 3(a), the PPI network consisted of 1859 nodes and 33869 edges, which represented 1859 interacting protein and 33869 interactions. According to the previous research, when the degree of all nodes was 40 , which was twice as large as the median degree, targets with significant functions can be screened [26]. Then, as shown in Figure 3(b), the further filtered PPI network consisting of 521 nodes and 15710 edges was constructed. In this network, the median parameters of DC, BC, CC, EC, LAC, and NC were 50, 206.609, 0.521042, $0.032009,12.66667$, and 14.0218 , respectively. The above data can be used as new reference standards for further screening, so the targets were determined with $\mathrm{DC}>50$, BC > 206.609, $\quad$ CC $>0.521042, \quad$ EC $>0.032009, \quad$ LAC $>$ 12.66667, and $\mathrm{NC}>14.0218$ (Figure 3(c)). After a series of topology analysis, a total of 65 core target genes were eventually confirmed for SXD against T2DM, which are likely to exert antidiabetic mechanisms through direct and indirect effects.

3.4. GO and KEGG Analyses. A total of the 37 common targets in SXD-treated T2DM obtained from the compoundtarget network (Figure 2) were used to perform GO and KEGG pathway analyses. And the DAVID database was used during the data processing. Among them, the candidate targets for GO enrichment analysis were carried out in three different biological aspects, namely, biological process, cellular component, and molecular function. There were 172 enrichment GO terms with FDR $<0.05$, including 387 in biological process, 23 in cellular component, and 40 in molecular function. The top 20 terms among the enriched entries were shown in Figure 4, of which there are 26 genes in biological process, 25 genes in cellular component, and 21 genes in molecular function. Through the analysis of enriched GO items, these genes distributed in biological processes, cellular components, and molecular functions were mainly manifested in the regulation of oxygen levels, regulation of 
TABLE 1: Active compounds and ADME parameters of Sanhuang Xiexin decoction (SXD).

\begin{tabular}{|c|c|c|c|c|c|c|c|c|c|}
\hline ID & Name & OB & $\mathrm{DL}$ & Source & ID & Name & $\mathrm{OB}$ & $\mathrm{DL}$ & Source \\
\hline MOL002235 & Eupation & 50.8 & 0.41 & Dahuang & MOL002908 & $\begin{array}{l}\text { 5,8,2' -Trihydroxy-7- } \\
\text { methoxyflavone }\end{array}$ & 37.01 & 0.27 & Huangqin \\
\hline MOL002251 & Mutatochrome & 48.64 & 0.61 & Dahuang & MOL002909 & $\begin{array}{l}\text { 5,7,2,5-Tetrahydroxy- } 8 \\
\text { 6-dimethoxyflavone }\end{array}$ & 33.82 & 0.45 & Huangqin \\
\hline MOL002259 & Physciondiglucoside & 41.65 & 0.63 & Dahuang & MOL002910 & Carthamidin & 41.15 & 0.24 & Huangqin \\
\hline MOL002260 & Procyanidin B-5, $3^{\prime}$-O-gallate & 31.99 & 0.32 & Dahuang & MOL002911 & $\begin{array}{c}2,6,2^{\prime}, 4^{\prime} \text {-Tetrahydroxy- } \\
6^{\prime} \text {-methoxychaleone }\end{array}$ & 69.04 & 0.22 & Huangqin \\
\hline MOL002268 & Rhein & 47.07 & 0.28 & Dahuang & MOL002913 & Dihydrobaicalin_qt & 40.04 & 0.21 & Huangqin \\
\hline MOL002276 & Sennoside E_qt & 50.69 & 0.61 & Dahuang & MOL002914 & Eriodyctiol (flavanone) & 41.35 & 0.24 & Huangqin \\
\hline MOL002280 & $\begin{array}{l}\text { Torachrysone-8-O-beta-D- } \\
\quad\left(6^{\prime} \text {-oxayl)-glucoside }\right.\end{array}$ & 43.02 & 0.74 & Dahuang & MOL002915 & Salvigenin & 49.07 & 0.33 & Huangqin \\
\hline MOL002281 & Toralactone & 46.46 & 0.24 & Dahuang & MOL002917 & $\begin{array}{l}5,2^{\prime}, 6^{\prime} \text {-Trihydroxy-7,8- } \\
\text { dimethoxyflavone }\end{array}$ & 45.05 & 0.33 & Huangqin \\
\hline MOL002288 & $\begin{array}{l}\text { Emodin-1-O-beta-D- } \\
\text { glucopyranoside }\end{array}$ & 44.81 & 0.8 & Dahuang & MOL002925 & $\begin{array}{c}5,7,2^{\prime}, 6^{\prime}- \\
\text { Tetrahydroxyflavone }\end{array}$ & 37.01 & 0.24 & Huangqin \\
\hline MOL002293 & Sennoside D_qt & 61.06 & 0.61 & Dahuang & MOL002926 & Dihydrooroxylin A & 38.72 & 0.23 & Huangqin \\
\hline MOL002297 & Daucosterol_qt & 35.89 & 0.7 & Dahuang & MOL002927 & Skullcapflavone II & 69.51 & 0.44 & Huangqin \\
\hline MOL002303 & Palmidin A & 32.45 & 0.65 & Dahuang & MOL002928 & Oroxylin a & 41.37 & 0.23 & Huangqin \\
\hline MOL000358 & Beta-sitosterol & 36.91 & 0.75 & Dahuang & MOL002932 & Panicolin & 76.26 & 0.29 & Huangqin \\
\hline MOL000471 & Aloe-emodin & 83.38 & 0.24 & Dahuang & MOL002933 & $\begin{array}{l}5,7,4^{\prime} \text {-Trihydroxy-8- } \\
\text { methoxyflavone }\end{array}$ & 36.56 & 0.27 & Huangqin \\
\hline MOL000554 & $\begin{array}{l}\text { Gallic acid-3-O-(6'-O- } \\
\text { galloyl)-glucoside }\end{array}$ & 30.25 & 0.67 & Dahuang & MOL002934 & Neobaicalein & 104.34 & 0.44 & Huangqin \\
\hline MOL000096 & (-)-Catechin & 49.68 & 0.24 & Dahuang & MOL002937 & Dihydrooroxylin & 66.06 & 0.23 & Huangqin \\
\hline MOL001454 & Berberine & 36.86 & 0.78 & Huanglian & MOL000358 & Beta-sitosterol & 36.91 & 0.75 & Huangqin \\
\hline MOL013352 & Obacunone & 43.29 & 0.77 & Huanglian & MOL000359 & Sitosterol & 36.91 & 0.75 & Huangqin \\
\hline MOL002894 & Berberrubine & 35.74 & 0.73 & Huanglian & MOL000525 & Norwogonin & 39.4 & 0.21 & Huangqin \\
\hline MOL002897 & Epiberberine & 43.09 & 0.78 & Huanglian & MOL000552 & $\begin{array}{l}5,2^{\prime} \text {-Dihydroxy- } 6,7,8- \\
\text { trimethoxyflavone }\end{array}$ & 31.71 & 0.35 & Huangqin \\
\hline MOL002903 & (R)-canadine & 55.37 & 0.77 & Huanglian & MOL000073 & Ent-epicatechin & 48.96 & 0.24 & Huangqin \\
\hline MOL002904 & Berlambine & 36.68 & 0.82 & Huanglian & MOL000449 & Stigmasterol & 43.83 & 0.76 & Huangqin \\
\hline MOL002907 & Corchoroside A_qt & 104.95 & 0.78 & Huanglian & MOL001458 & Coptisine & 30.67 & 0.86 & Huangqin \\
\hline MOL000622 & Magnograndiolide & 63.71 & 0.19 & Huanglian & MOL001490 & $\begin{array}{c}\text { bis }[(2 S)-2 \text {-Ethylhexyl] } \\
\text { benzene-1,2-dicarboxylate }\end{array}$ & 43.59 & 0.35 & Huangqin \\
\hline MOL000762 & Palmidin A & 35.36 & 0.65 & Huanglian & MOL001506 & Supraene & 33.55 & 0.42 & Huangqin \\
\hline MOL000785 & Palmatine & 64.6 & 0.65 & Huanglian & MOL002879 & Diop & 43.59 & 0.39 & Huangqin \\
\hline MOL000098 & Quercetin & 46.43 & 0.28 & Huanglian & MOL002897 & Epiberberine & 43.09 & 0.78 & Huangqin \\
\hline MOL001458 & Coptisine & 30.67 & 0.86 & Huanglian & MOL008206 & Moslosooflavone & 44.09 & 0.25 & Huangqin \\
\hline MOL002668 & Worenine & 45.83 & 0.87 & Huanglian & MOL010415 & $\begin{array}{l}\text { 11,13-Eicosadienoic acid, } \\
\text { methyl ester }\end{array}$ & 39.28 & 0.23 & Huangqin \\
\hline MOL008647 & Moupinamide & 86.71 & 0.26 & Huanglian & MOL012245 & $\begin{array}{l}\text { 5,7,4' - Trihydroxy-6- } \\
\text { methoxyflavanone }\end{array}$ & 36.63 & 0.27 & Huangqin \\
\hline MOL001689 & Acacetin & 34.97 & 0.24 & Huangqin & MOL012246 & $\begin{array}{l}\text { 5,7,4'-Trihydroxy- } 8 \text { - } \\
\text { methoxyflavanone }\end{array}$ & 74.24 & 0.26 & Huangqin \\
\hline MOL000173 & Wogonin & 30.68 & 0.23 & Huangqin & MOL012266 & Rivularin & 37.94 & 0.37 & Huangqin \\
\hline MOL000228 & $\begin{array}{l}\text { (2R)-7-Hydroxy-5-methoxy- } \\
\text { 2-phenylchroman-4-one }\end{array}$ & 55.23 & 0.2 & Huangqin & MOL002935 & Baicalin & 29.53 & 0.77 & Huangqin \\
\hline MOL002714 & Baicalein & 33.52 & 0.21 & Huangqin & & & & & \\
\hline
\end{tabular}




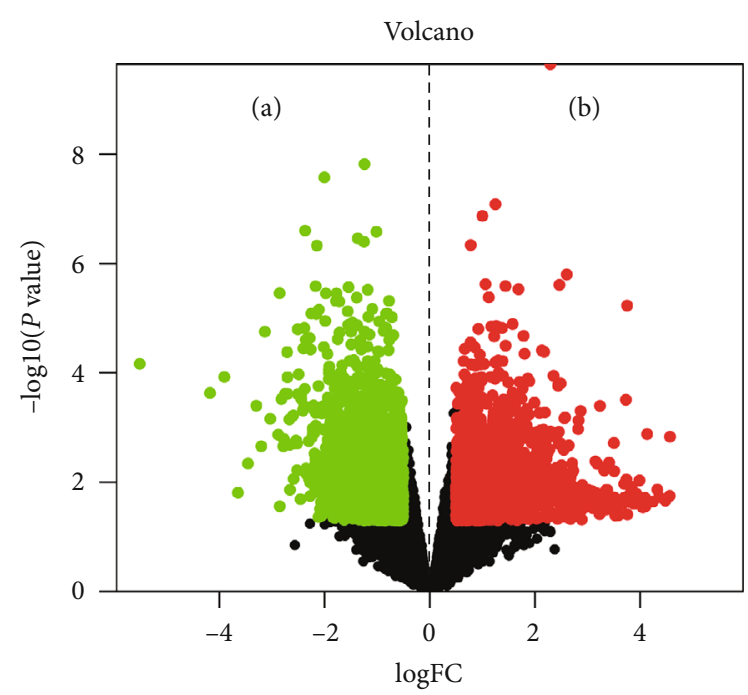

Figure 1: Volcano map of the differential genes. The abscissa represents $\log \mathrm{FC}$, which is the fold change in the gene expression. The ordinate represents $-\log 10(P$ value $)$, which is the statistical significance of the change in gene expression. The green dots on (a) represent downregulated genes, and the red dots on (b) represent upregulated genes.

serine/threonine kinase activity, mitochondria, platelet activation, and protein kinase regulator activity.

Similarly, we also performed KEGG analysis to determine which pathways exert an enormous function on the pathological mechanism. As shown in Figure 5, the top 20 significantly enriched pathways $($ FDR $<0.05)$ were identified, in which there were PI3K-Akt signaling pathway, cancers, HIF-1 signaling pathway, JAK-STAT signaling pathway, endocrine resistance, and AGE-RAGE signaling pathway in diabetic complications.

3.5. Gene-Pathway Network Analysis. In order to further screen the key genes enriched in the significant pathways, a gene-pathway network was established using Cytoscape software (Figure 6). Degree centrality (DC) was used to perform the topological analysis of the twenty pathways and twentyone genes obtained in the KEGG analysis (Figure 5). In the gene-pathway network, the $\mathrm{V}$ shapes represent pathways and the diamond shapes represent the screened target genes. Through the visualized network, it can be intuitively seen that CDKN1A had the largest DC and was the core gene in the entire diagram. At the same time, several other genes, such as CCND1, RAF1, ERBB2, EGF, and VEGFA, also had larger DC. And all of them were the key target genes for SXD against T2DM.

\section{Discussion}

T2DM is an endocrine disease with clinical manifestations of metabolic disorders and chronic complications, and its occurrence is related to a variety of factors such as heredity, lifestyle, biochemistry, posture, and environment. The pathogenesis of T2DM mainly involves islet $\beta$-cell dysfunction, insulin resistance, inflammatory response, and oxidative stress response. SXD was an effective common prescription in the treatment of T2DM in TCM, which has demonstrated significant clinical effects in TCM clinical practice. According to previous studies, Huanglian, Huangqin, and Dahuang three components in SXD have antidiabetic effects. The active compounds in Huanglian can improve glucose metabolism, pancreatic beta cells, and insulin resistance and modulate the gut microbiota [27]. The active compounds in Huangqin can improve glucose metabolism and insulin sensitivity in peripheral tissue [28]. The active compounds in Dahuang can improve glucose metabolism and $\beta$-cell function, modulate the gut microbiota, and reduce oxidative stress and inflammation $[29,30]$. However, it is not clear how SXD works in the antidiabetic pathological processes. Therefore, we use the method of network pharmacology to clarify the mechanism of active ingredients in SXD acting on T2DM.

In this paper, the compound-target network suggested that some compounds of SXD affected most targets. For instance, quercetin, aloe-emodin, and stigmasterol acted on 25,6 , and 5 targets, respectively. Consequently, they may probably be vital multifunctional active compounds of SXD against T2DM. It is well known that both quercetin [31-33] and aloe-emodin [34] exhibit a wide range of biological effects, including anti-inflammatory, antioxidant, anticancer, and especially the protective effect on T2DM. Youl et al. [35] reported that quercetin could significantly increase insulin secretion via ERK1/2 phosphorylation in INS-1 cells. And previous reports indicate that quercetin was able to promote wound healing of T2DM rats and improved glycemia by modulating inflammation and oxidative stress [36]. Besides, studies have shown that aloe-emodin can effectively treat $\beta$ cell failure in T2DM patients, because it can prevent the occurrence of pancreatic $\beta$-cell sugar glucotoxicity by regulating proinflammatory cytokines (IFN- $\gamma$, and IL1 $\beta$ ) [37]. Similarly, stigmasterol is a member of the plant sterol family and has multiple pharmacological effects, such as antiosteoarthritis, anti-inflammatory, and antitumor properties. [38-41] One recent study showed that an extract from banana containing $21.91 \%$ stigmasterol exhibited a potential antidiabetic effect in alloxan-induced diabetic rats [42]. It can be seen that quercetin, aloe-emodin, and stigmasterol have significant antidiabetic effects in T2DM. And they all have high oral bioavailability and are considered to be representative compounds in SXD.

As shown in Figure 3, in order to get more accurate genes related to T2DM, a series of parameters were performed to screen for the key targets in the PPI network. Eventually, 65 significant targets were determined, which may play an important role in the pathophysiology of T2DM through direct or indirect effects. Among these genes, the top 5 genes were YWHAZ, HNRNPA1, HSPA8, HSP90AA1, and HSPA5, respectively. One research reported that YWHAZ mRNA and protein levels are significantly upregulated in the placental tissues of gestational diabetes mellitus (GDM) patients due to the downregulation of miR-214 [43]. The result indicates that elevated expression of YWHAZ protein played a regulatory role in the occurrence of GDM. 


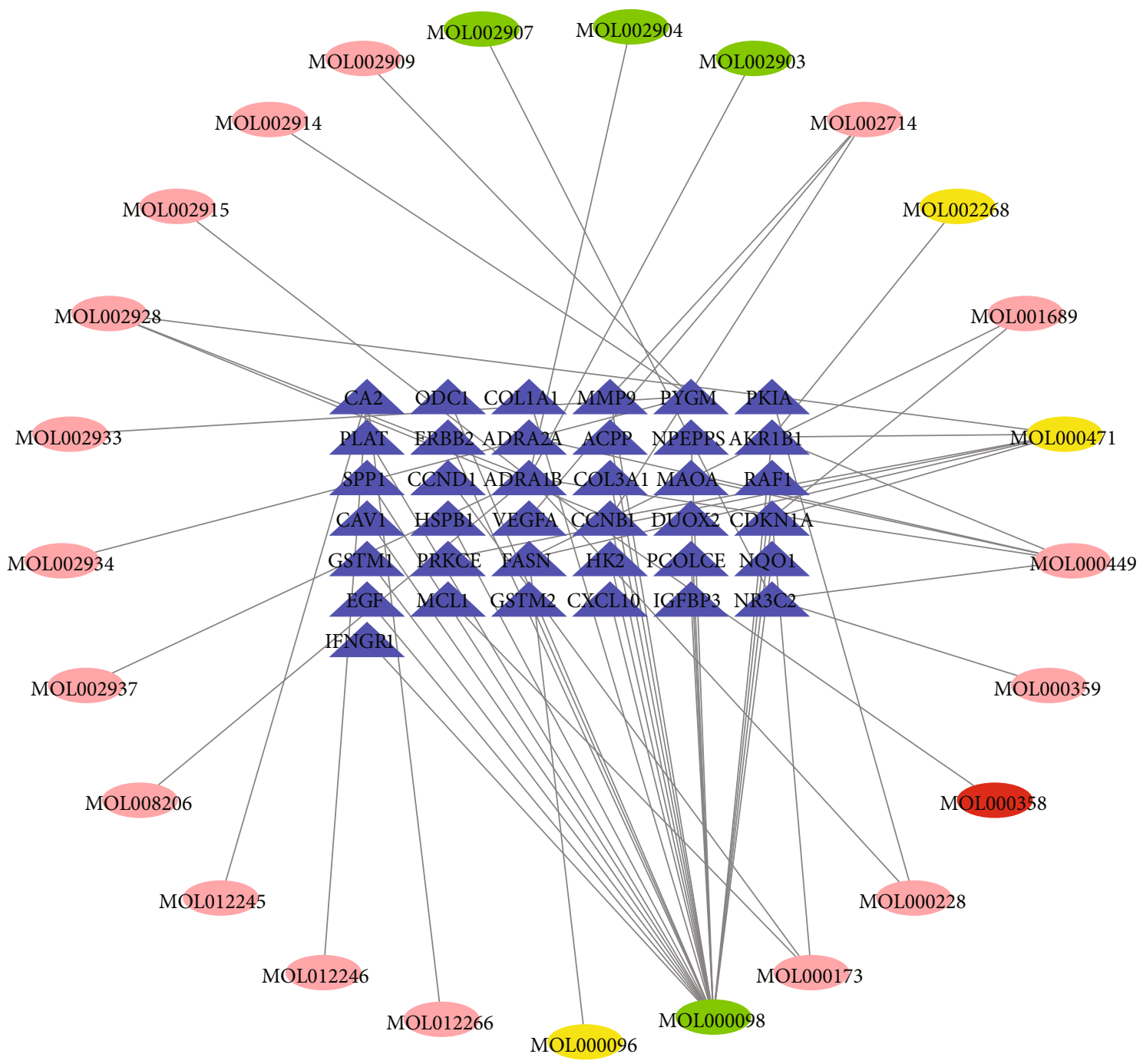

Figure 2: Compound-target network: the blue triangles represent targets related to T2DM in SXD; the yellow, green, and pink ovals represent the compounds from Dahuang, Huanglian, and Huangqin, respectively. The red oval represents a common component from Dahuang and Huangqin.

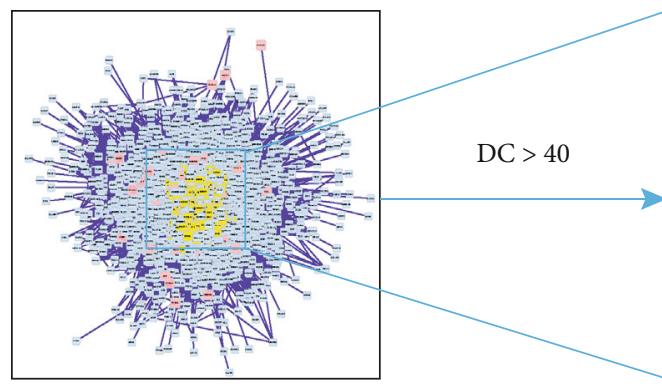

(A)

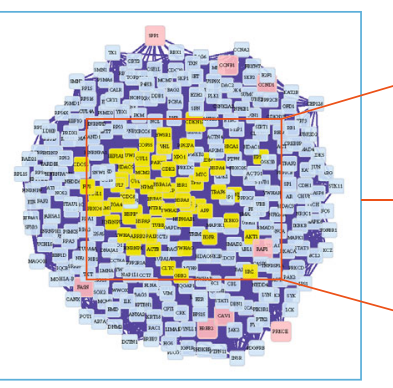

(B)

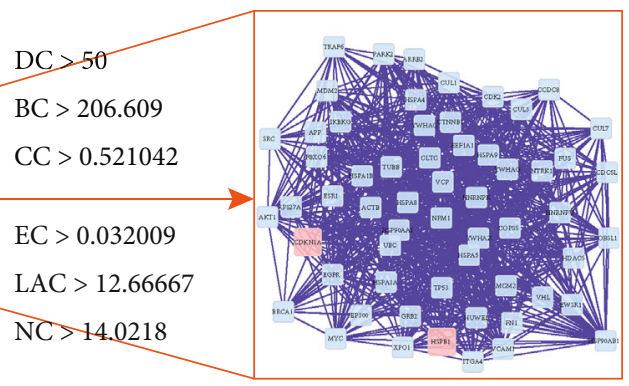

(C)

FIGURE 3: PPI network. (a) The combined PPI network of SXD-related targets and T2DM-related targets. (b) PPI network with important targets extracted from (a). (c) PPI network with core targets extracted from (b).

HNRNPA1 maintains the stability of blood glucose in T2DM by regulating the sensitivity of insulin in muscle cells. A study by Zhao et al. [44]. showed that the metabolic capacity and insulin sensitivity of patients with T2DM are worsened by reduced expression of skeletal muscle HNRNPA1 because it inhibits glycogen synthesis in the body.

Heat-shock proteins (HSPs) can protect cells from oxidative stress, inflammation, and apoptosis because they can 


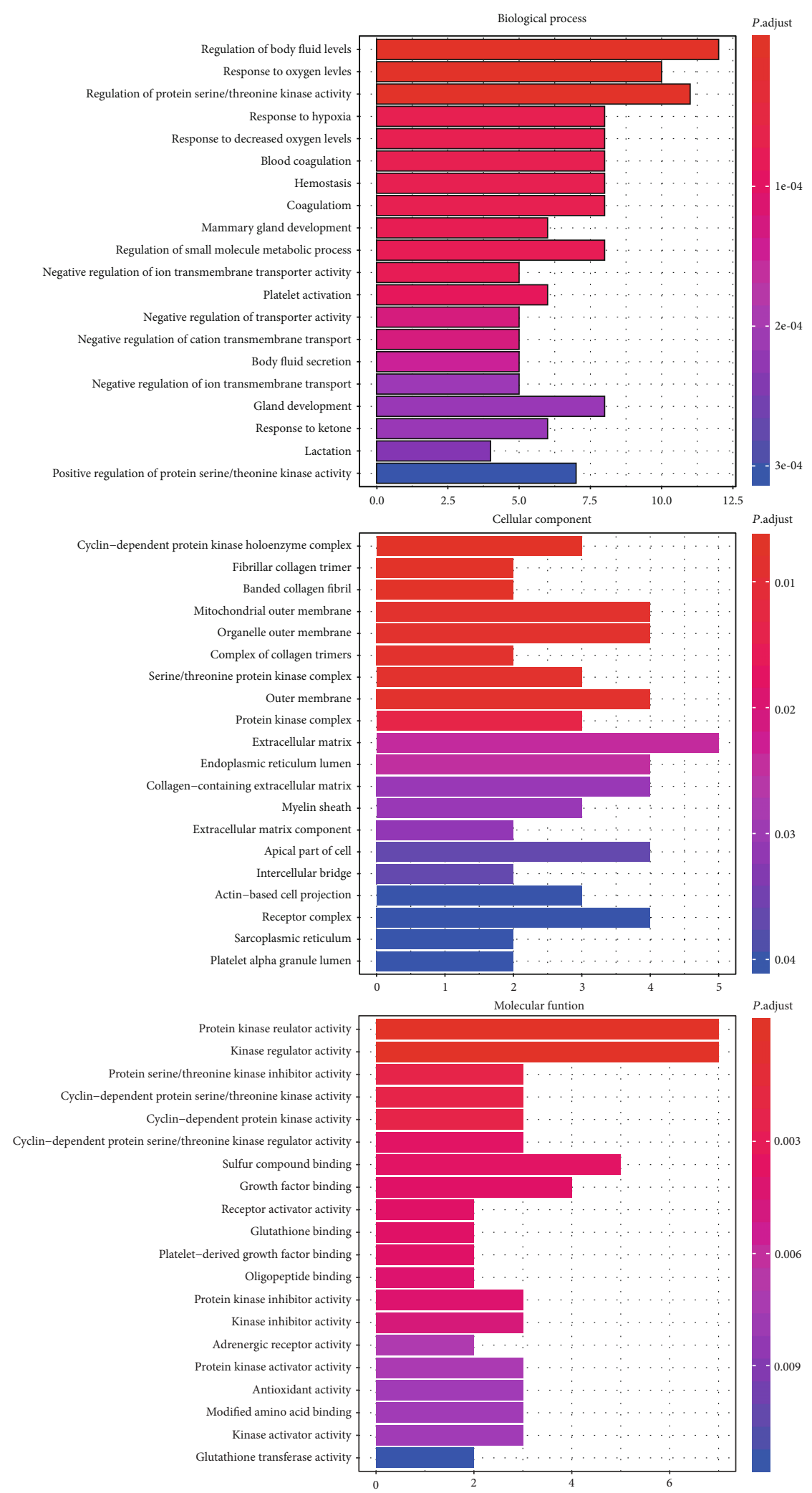

Figure 4: GO enrichment analysis. The top $20 \mathrm{GO}$ enriched terms with FDR $<0.05$ were screened. The abscissa represents the $P$ adjust value of the enriched terms and the ordinate represents the name of the enriched terms.

counteract the deleterious effects of hyperglycemia in the target organs of diabetes vascular complications [45]. Changes in the expression of related HSPs (HSPA8 [46],
HSP90AA1 [47], and HSPA5 [48],) have been confirmed in complications of diabetes and are functionally related to hyperglycemia-induced cell damage. Therefore, the above 


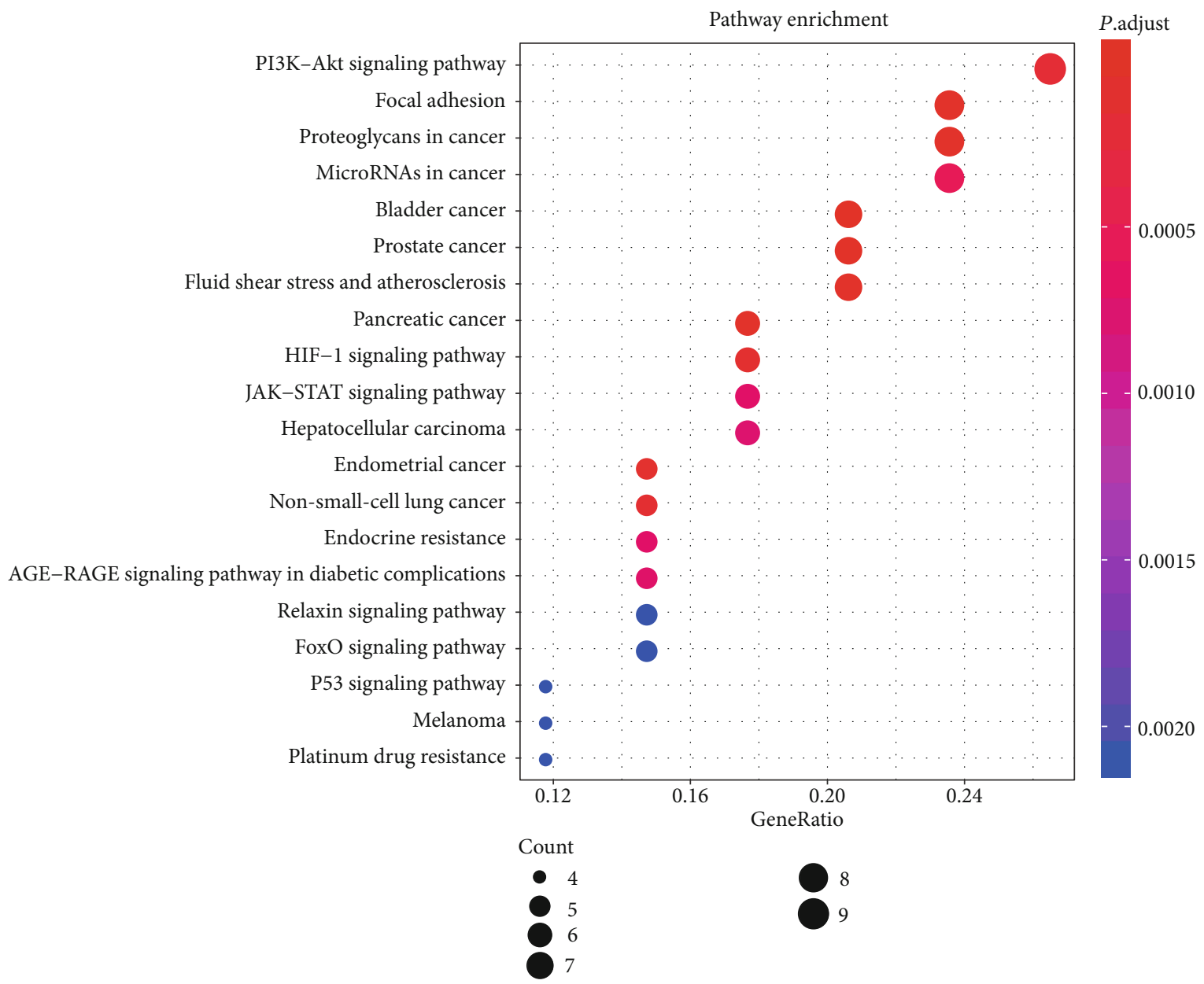

FIGURE 5: KEGG pathway enrichment analysis. The top 20 significant pathways with FDR $<0.05$ were identified. The abscissa represents the GeneRatio of the enriched genes and the ordinate represents the name of the enriched pathways. The size of the dots represents the number of genes, and the color represents the significance of the FDR value.

five genes may have the potential to serve as valuable clinical biomarkers and provide new directions for the treatment of T2DM.

The common targets of SXD against T2DM were used to perform biological processes, cellular components, and molecular function analysis. In the biological processes, there are enriched terms of regulation of body fluid level, response to oxygen levels, regulation of protein serine/threonine kinase activity, hypoxia, and platelet activation (Figure 4). Different levels of body fluid level may play an important role in determining morphologic features that appear in diabetic macular edema (DME). Studies have pointed out that body fluid levels are related to the severity of diabetic retinopathy (DR) and that volume overload is an independent risk factor for it [49]. Patients with T2DM are easily prone to be in a state of hypoxia because of the increase in glycated hemoglobin levels which result in the weakened capacity of releasing oxygen into the plasma. And a study of Mason et al. found evidence that skeletal muscle oxygenation during exercise was more impaired in T2DM patients than in overweight and sedentary controls [50-53]. Protein kinases also exhibited an important role in the progression of T2DM. Some data suggest that STK11 can improve the lipotoxicity of islet cells and the glucose regulation of insulin secretion by activating the LKB1-AMPK pathway, regulating the secretion of insulin-like growth factor 1 (IGF-1) to improve IR in T2DM patients [54-56]. In addition, due to the unique inflammatory environment under diabetic condition, platelets are more prone to adhesion to endothelial cells and can be used as a sensitive biomarker for T2DM with vascular diseases [57]. Besides, these biological processes were also related to cellular components and molecular function, including collagen trimer, mitochondrial, endoplasmic reticulum, growth factor, and glutathione binding. As shown by some studies, ultrastructural abnormalities in cells of T2DM patients have also been found, such as cytoplasmic vacuolization, mitochondrial swelling, abnormal chromatin condensation, and endoplasmic reticulum expansion.

In the KEGG analysis, the top 20 KEGG pathways were significantly enriched including the PI3K-Akt signaling pathway, cancers, HIF-1 signaling pathway, JAK-STAT signaling pathway, endocrine resistance, and AGE-RAGE signaling pathway in diabetic complications. The PI3K-Akt signaling pathway was the most prominently enriched term. In T2DM, insulin is the major ligand that regulates metabolism in the PI3K/AKT pathway. Thus, the PI3K-Akt signaling 


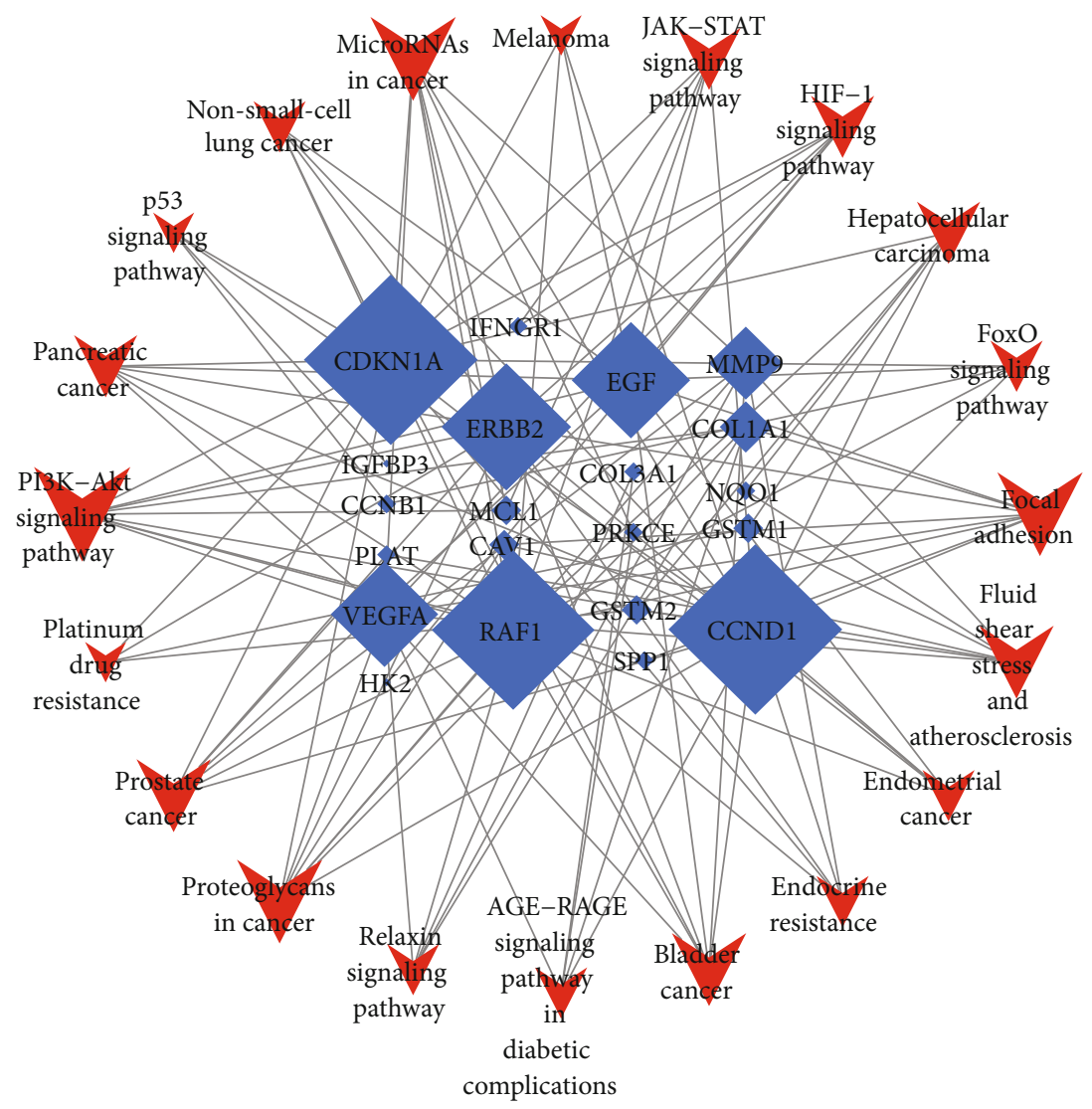

Figure 6: Gene-pathway network. Degree centrality (DC) was used to perform the topological analysis of the 20 pathways and 21 genes. The blue diamonds represent candidate genes and the red V-shapes represent pathways. The size of nodes represents the value of degree centrality.

pathway is activated by secreted insulin after eating, which increases glucose utilization and reduces gluconeogenesis in the liver and muscle [58]. Besides, the PI3K-Akt signaling can also exhibit its importance by mediating growth factor signals in glucose homeostasis, lipid metabolism, and protein synthesis [59]. Hypoxia is a pathological state in the cells of T2DM patients, and the HIF-1 signaling pathway plays a complex role in this process [60]. There is one clue that HIF $1 \alpha$ activation can exacerbate the inflammation and fibrosis status of T2DM during hypoxia, thereby elevating obesity and insulin resistance in mice [61]. The JAK/STAT pathway is known to be involved in the inflammatory response and is one of the key ways of cytokine signal transduction. Inhibition of JAK phosphorylation and activation can prevent phosphorylation and activation of STATs, thereby alleviating insulin resistance because of reduced production of cytokines [62]. Studies have shown that the deficiency of STAT4 in high-fat diet-fed mice in T2DM mice was associated with reduced adipose tissue inflammation and decreased insulin resistance [63]. In this study, several pathways related to cancer were also significantly enriched (Figure 5). The association between T2DM and cancer may be explained in part by some shared risk factors such as aging, obesity, and diet [64]. In addition, the notion that glucose metabolism and cancer are associated can also be speculated in relation to the antidiabetes drug metformin, which has reported associations with reduced risk of cancer [65]. In addition, SXD may function by regulating other pathways, including endocrine resistance, AGE-RAGE signaling pathway, FoxO signaling pathway, and P53 signaling pathway. Among these, the FoxO pathway is one of those key factors in the transition from IR to the damage of islet $\beta$-cells [66].

Gene-pathway network was established to investigate core target genes for SXD against T2DM. The network showed that CDKN1A had the largest DC, which may be the core target gene. Other top five genes were selected as key target genes, namely, CCND1, RAF1, ERBB2, EGF, and VEGFA. CDKN1A is a substance induced by tumor suppressor P53, which is needed in G1-S blocking of DNA damaged cells. Its main role is to prevent apoptosis, maintain beta cell quality, and participate in DNA repair after DNA damage [67]. Studies of gene sequencing from human islets with and without T2DM have shown that both CDKN1A and CCND1 are highly expressed in diabetic patients [68].. RAF1 kinase specifically works to control cell proliferation to maintain the quantity of pancreatic beta cells [69]. In T2DM patients, lower RAF1 kinase levels were found to have a negative effect on the proliferation of pancreatic beta cell [70-72]. ERBB2 has shown close associations with molecules governing lipid metabolism [73] and impaired glucose metabolism in T2DM [74]. Additionally, a cross-sectional study by Memon et al. [75] showed a significant association between ERBB2 and hyperglycemia and insulin resistance. EGFR can be abnormally activated during diabetes or 
atherosclerosis [76] and the enhanced EGFR activity with downstream endoplasmic reticulum (ER) stress in cardiovascular system has been reported in T2DM [77, 78]. VEGFA is found to be most relevant to diabetic retinopathy (DR) in the VEGF family. $\mathrm{Xu}$ et al. [79] have found that upregulated VEGFA can promote the development of DR via the low expression of microRNA-15b in retinal capillary endothelial cells and pericytes of diabetic rats.

\section{Conclusion}

In this study, the network pharmacological approaches were used to elucidate the mechanisms and molecular targets of SXD on T2DM. Quercetin, aloe-emodin, and stigmasterol in SXD can regulate most targets related to T2DM. Through the PPI network screening, it was found that 5 key genes (YWHAZ, HNRNPA1, HSPA8, HSP90AA1, and HSPA5) obtained through protein interactions may provide new ideas for the treatment of diabetes. SXD may regulate an antidiabetic function through the specific biological processes including regulation of body fluid level, response to oxygen levels, regulation of protein serine/threonine kinase activity, hypoxia, and platelet activation. The above functions are mainly performed through the PI3K-AKT signaling pathway, HIF-1 signaling pathway, JAK-STAT signaling pathway, and some other cancer pathways. And CDKN1A, CCND1, RAF1, ERBB2, EGF, and VEGFA in common targets were the key targets of active components in SXD against T2DM. Although we have tried to study the role of SXD in the treatment of T2DM through network pharmacology as far as possible, further experiments in vivo and in vitro are still needed to validate these views.

\section{Data Availability}

My data is obtained through authoritative database and software analysis. I can guarantee its reliability.

\section{Conflicts of Interest}

The authors declare that they have no conflicts of interest.

\section{Acknowledgments}

This research was funded by the National Natural Science Foundation of China (No. 81573790 and No. 81803829).

\section{References}

[1] K. Ogurtsova, J. D. da Rocha Fernandes, Y. Huang et al., "IDF diabetes atlas: global estimates for the prevalence of diabetes for 2015 and 2040," Diabetes Research and Clinical Practice, vol. 128, pp. 40-50, 2017.

[2] C. Bailey, "The current drug treatment landscape for diabetes and perspectives for the future," Clinical Pharmacology \& Therapeutics, vol. 98, no. 2, pp. 170-184, 2015.

[3] A. Y. Y. Cheng and I. G. Fantus, "Oral antihyperglycemic therapy for type 2 diabetes mellitus," Canadian Medical Association Journal, vol. 172, no. 2, pp. 213-226, 2005.
[4] Q. Zhang, R. Li, W. Peng et al., "Identification of the active constituents and significant pathways of Guizhi-ShaoyaoZhimu decoction for the treatment of diabetes mellitus based on molecular docking and network pharmacology," Combinatorial Chemistry \& High Throughput Screening, vol. 22, no. 9, pp. 584-598, 2019.

[5] D. He, J.-h. Huang, Z.-y. Zhang et al., "A network pharmacology-based strategy for predicting active ingredients and potential targets of LiuWei DiHuang pill in treating type 2 diabetes mellitus," Drug Design, Development and Therapy, vol. 13, pp. 3989-4005, 2019.

[6] S. Di, L. Han, Q. Wang et al., "A network pharmacology approach to uncover the mechanisms of Shen-Qi-Di-Huang decoction against diabetic nephropathy," Evidence-Based Complementary and Alternative Medicine, vol. 2018, Article ID 7043402, 14 pages, 2018.

[7] S. Sun and Y. Lin, Bei ji qian jin yao fang[M], Wu xian hsu min pu ji, 1878.

[8] B. Wu, M. Sui, Y. Zhu, K. Ding, and Y. F. Fan, "Forty-three cases of type II diabetes mellitus with phlegm-dampness-heat accumulation syndrome treated with three yellows decoction," Henan Traditional Chinese Medicine, vol. 39, no. 6, pp. 839842, 2019.

[9] P. Bing, G. Jing, Z. Linhua, Z. Xiyan, Z. Qiang, and T. Xiaolin, "Retrospective study of traditional Chinese medicine treatment of type 2 diabetes mellitus," Journal of Traditional Chinese Medicine, vol. 36, no. 3, pp. 307-313, 2016.

[10] Z. Z. Zhu, M. Q. Xiong, and A. Z. Lin, "Effect of sanhuang jiangtang recipe on insulin peripheral resistance in type II diabetics," Zhongguo Zhong xi yi jie he za zhi Zhongguo Zhongxiyi jiehe zazhi $=$ Chinese journal of integrated traditional and Western medicine / Zhongguo Zhong xi yi jie he xue hui, Zhongguo Zhong yi yan jiu yuan zhu ban, vol. 17, no. 10, p. 590, 1997.

[11] Y.-T. Shih, I.-J. Chen, Y.-C. Wu, and Y.-C. Lo, "San-HuangXie-Xin-Tang Protects against Activated Microglia- and 6OHDA- Induced Toxicity in Neuronal SH-SY5Y Cells," Evidence-based Complementary and Alternative Medicine, vol. 2011, Article ID 429384, 11 pages, 2011.

[12] Y. Saegusa, A. Sugiyama, A. Takahara, Y. Nagasawa, and K. Hashimoto, "Relationship between phosphodiesterase inhibition induced by several Kampo medicines and smooth muscle relaxation of gastrointestinal tract tissues of rats," Journal of Pharmacological Sciences, vol. 93, no. 1, pp. 6268, 2003.

[13] S.-F. Liou, J.-H. Hsu, J.-C. Liang et al., "San-Huang-Xie-XinTang protects cardiomyocytes against hypoxia/reoxygenation injury via inhibition of oxidative stress-induced apoptosis," Journal of Natural Medicines, vol. 66, no. 2, pp. 311-320, 2012.

[14] Y.-S. Wang, R.-T. Lin, H.-Y. Cheng, S. F. Yang, W. W. Chou, and S. H. H. Juo, "Anti-atherogenic effect of San-Huang-XieXin-Tang, a traditional Chinese medicine, in cultured human aortic smooth muscle cells," Journal of Ethnopharmacology, vol. 133, no. 2, pp. 442-447, 2011.

[15] J. S. Wu, R. Shi, X. Lu, Y. M. Ma, and N. N. Cheng, "Combination of active components of Xiexin decoction ameliorates renal fibrosis through the inhibition of NF- $\kappa \mathrm{B}$ and TGF$\beta 1 /$ Smad pathways in $\mathrm{db} / \mathrm{db}$ diabetic mice," PLoS One, vol. 10, no. 3, article e0122661, 2015.

[16] Y. Jiang, N. Liu, S. Zhu, X. Hu, D. Chang, and J. Liu, "Elucidation of the mechanisms and molecular targets of Yiqi Shexue formula for treatment of primary immune thrombocytopenia 
based on network pharmacology," Frontiers in Pharmacology, vol. 10, 2019.

[17] S. D. Navaneethan, S. Aloudat, and S. Singh, "A systematic review of patient and health system characteristics associated with late referral in chronic kidney disease," BMC Nephrology, vol. 9, no. 1, p. 3, 2008.

[18] X. Xu, W. Zhang, C. Huang et al., "A novel chemometric method for the prediction of human oral bioavailability," International Journal of Molecular Sciences, vol. 13, no. 6, pp. 6964-6982, 2012.

[19] M. Hong, S. Li, H. Y. Tan et al., "A network-based pharmacology study of the herb-induced liver injury potential of traditional hepatoprotective Chinese herbal medicines," Molecules, vol. 22, no. 4, p. 632, 2017.

[20] J. Ru, P. Li, J. Wang et al., "TCMSP: a database of systems pharmacology for drug discovery from herbal medicines," Journal of Cheminformatics, vol. 6, no. 1, p. 13, 2014.

[21] D. S. Wishart, Y. D. Feunang, A. C. Guo et al., "DrugBank 5.0: a major update to the DrugBank database for 2018," Nucleic Acids Research, vol. 46, pp. D1074-D1082, 2018.

[22] P. Shannon, A. Markiel, O. Ozier et al., "Cytoscape: a software environment for integrated models of biomolecular interaction networks," Genome Research, vol. 13, no. 11, pp. 24982504, 2003.

[23] A. Martin, M. E. Ochagavia, L. C. Rabasa, J. Miranda, J. Fernandez-de-Cossio, and R. Bringas, "BisoGenet: a new tool for gene network building, visualization and analysis," $B M C$ Bioinformatics, vol. 11, no. 1, p. 91, 2010.

[24] D. W. Huang, B. T. Sherman, and R. A. Lempicki, "Systematic and integrative analysis of large gene lists using DAVID bioinformatics resources," Nature Protocols, vol. 4, no. 1, pp. 44-57, 2009.

[25] V. S. Rao, K. Srinivas, G. N. Sujini, and G. N. S. Kumar, "Protein-protein interaction detection: methods and analysis," International Journal of Proteomics, vol. 2014, Article ID 147648, 12 pages, 2014.

[26] Y. Zhang, Z. Li, M. Yang et al., "Identification of GRB2 and GAB1 coexpression as an unfavorable prognostic factor for hepatocellular carcinoma by a combination of expression profile and network analysis," PLoS One, vol. 8, no. 12, article e85170, 2013.

[27] J. Wang, L. Wang, G.-H. Lou et al., "Coptidis rhizoma: a comprehensive review of its traditional uses, botany, phytochemistry, pharmacology and toxicology," Pharmaceutical Biology, vol. 57, no. 1, pp. 193-225, 2019.

[28] P. Fang, M. Yu, M. Shi, P. Bo, X. Gu, and Z. Zhang, "Baicalin and its aglycone: a novel approach for treatment of metabolic disorders," Pharmacological Reports, vol. 72, no. 1, pp. 13-23, 2020.

[29] F.-R. Cheng, H.-X. Cui, J.-L. Fang, K. Yuan, and Y. Guo, “Ameliorative effect and mechanism of the purified anthraquinoneglycoside preparation from Rheum palmatum L. on type 2 diabetes mellitus," Molecules, vol. 24, no. 8, article 1454, 2019.

[30] H.-X. Cui, L.-S. Zhang, Y. Luo, K. Yuan, Z.-Y. Huang, and Y. Guo, "A purified anthraquinone-glycoside preparation from rhubarb ameliorates type 2 diabetes mellitus by modulating the gut microbiota and reducing inflammation," Frontiers in Microbiology, vol. 10, p. 1423, 2019.

[31] D. W. Lamson and M. S. Brignall, "Antioxidants and cancer, part 3: quercetin," Alternative Medicine Review: A Journal of Clinical Therapeutic, vol. 5, no. 3, pp. 196-208, 2000.
[32] A. W. Boots, G. R. M. M. Haenen, and A. Bast, "Health effects of quercetin: from antioxidant to nutraceutical," European Journal of Pharmacology, vol. 585, no. 2-3, pp. 325-337, 2008.

[33] M. Vessal, M. Hemmati, and M. Vasei, "Antidiabetic effects of quercetin in streptozocin-induced diabetic rats," Comparative Biochemistry and Physiology Part C: Toxicology \& Pharmacology, vol. 135, no. 3, pp. 357-364, 2003.

[34] X. Dong, Y. Zeng, Y. Liu et al., "Aloe-emodin: a review of its pharmacology, toxicity, and pharmacokinetics," Phytotherapy Research, vol. 34, no. 2, pp. 270-281, 2019.

[35] E. Youl, G. Bardy, R. Magous et al., "Quercetin potentiates insulin secretion and protects INS-1 pancreatic $\beta$-cells against oxidative damage via the ERK1/2 pathway," British Journal of Pharmacology, vol. 161, no. 4, pp. 799-814, 2010.

[36] O. M. Ahmed, T. Mohamed, H. Moustafa, H. Hamdy, R. R. Ahmed, and E. Aboud, "Quercetin and low level laser therapy promote wound healing process in diabetic rats via structural reorganization and modulatory effects on inflammation and oxidative stress," Biomedicine \& Pharmacotherapy, vol. 101, pp. 58-73, 2018.

[37] A. A. Alshatwi and P. Subash-Babu, "Aloe-emodin protects RIN-5F (pancreatic $\beta$-cell) cell from glucotoxicity via regulation of pro-inflammatory cytokine and downregulation of Bax and caspase 3," Biomolecules \& Therapeutics, vol. 24, no. 1, pp. 49-56, 2016.

[38] O. Gabay, C. Sanchez, C. Salvat et al., "Stigmasterol: a phytosterol with potential anti-osteoarthritic properties," Osteoarthritis and Cartilage, vol. 18, no. 1, pp. 106-116, 2010.

[39] S. Zielińska and A. Matkowski, "Phytochemistry and bioactivity of aromatic and medicinal plants from the genus Agastache (Lamiaceae)," Phytochemistry Reviews, vol. 13, no. 2, pp. 391416, 2014.

[40] J. J. Casal, M. Bollini, M. E. Lombardo, and A. M. Bruno, "Thalidomide analogues: tumor necrosis factor-alpha inhibitors and their evaluation as anti-inflammatory agents," European Journal of Pharmaceutical Sciences, vol. 83, pp. 114-119, 2016.

[41] Y.-S. Kim, X.-F. Li, K.-H. Kang, B. M. Ryu, and S. K. Kim, "Stigmasterol isolated from marine microalgae Navicula incerta induces apoptosis in human hepatoma HepG2 cells," BMB Reports, vol. 47, no. 8, pp. 433-438, 2014.

[42] R. Ramu, P. S. Shirahatti, S. Nayakavadi et al., "The effect of a plant extract enriched in stigmasterol and $\beta$-sitosterol on glycaemic status and glucose metabolism in alloxan-induced diabetic rats," Food \& Function, vol. 7, no. 9, pp. 39994011, 2016.

[43] X. Ma, Y. Jiang, L. Li, and X. H. Liu, "Upregulation of YWHAZ in placental tissues, blood, and amniotic fluid from patients with gestational diabetes mellitus related to downregulation of microRNA-214," International Journal of Clinical and Experimental Medicine, vol. 12, no. 8, pp. 9961-9968, 2019.

[44] M. Zhao, L. Shen, Z. Ouyang et al., "Loss of hnRNP A1 in murine skeletal muscle exacerbates high-fat diet-induced onset of insulin resistance and hepatic steatosis," Journal of Molecular Cell Biology, vol. 12, no. 4, pp. 277-290, 2020.

[45] S. Bellini, F. Barutta, R. Mastrocola, L. Imperatore, G. Bruno, and G. Gruden, "Heat shock proteins in vascular diabetic complications: review and future perspective," International Journal of Molecular Sciences, vol. 18, no. 12, p. 2709, 2017.

[46] Y. Liao and L. Tang, "The critical roles of HSC70 in physiological and pathological processes," Current Pharmaceutical Design, vol. 20, no. 1, pp. 101-107, 2014. 
[47] X. Yang, Y. Zhang, W. Xu et al., "Potential role of Hsp90 in rat islet function under the condition of high glucose," Acta Diabetologica, vol. 53, no. 4, pp. 621-628, 2016.

[48] Y. Zhao, Y. Yan, Z. Zhao, S. Li, and J. Yin, "The dynamic changes of endoplasmic reticulum stress pathway markers GRP78 and CHOP in the hippocampus of diabetic mice," Brain Research Bulletin, vol. 111, pp. 27-35, 2015.

[49] M.-J. Tsai, C.-K. Cheng, and Y.-C. Wang, "Association of body fluid expansion with optical coherence tomography measurements in diabetic retinopathy and diabetic macular edema," Investigative Ophthalmology \& Visual Science, vol. 60, no. 10, pp. 3606-3612, 2019.

[50] B. J. Behnke, C. A. Kindig, P. McDonough, D. C. Poole, and W. L. Sexton, "Dynamics of microvascular oxygen pressure during rest-contraction transition in skeletal muscle of diabetic rats," American Journal of Physiology-Heart and Circulatory Physiology, vol. 283, no. 3, pp. H926-H932, 2002.

[51] T. A. Bauer, J. E. B. Reusch, M. Levi, and J. G. Regensteiner, "Skeletal muscle deoxygenation after the onset of moderate exercise suggests slowed microvascular blood flow kinetics in type 2 diabetes," Diabetes Care, vol. 30, no. 11, pp. 28802885, 2007.

[52] S. Tagougui, E. Leclair, P. Fontaine et al., "Muscle oxygen supply impairment during exercise in poorly controlled type 1 diabetes," Medicine and Science in Sports and Exercise, vol. 47, no. 2, pp. 231-239, 2015.

[53] J. C. Frisbee, "Impaired dilation of skeletal muscle microvessels to reduced oxygen tension in diabetic obese Zucker rats," American Journal of Physiology-Heart and Circulatory Physiology, vol. 281, no. 4, pp. H1568-H1574, 2001.

[54] E. S. Buhl, N. Jessen, R. Pold et al., "Long-term AICAR administration reduces metabolic disturbances and lowers blood pressure in rats displaying features of the insulin resistance syndrome," Diabetes, vol. 51, no. 7, pp. 21992206, 2002.

[55] Y. Wang, E. Paulo, D. Wu et al., “Adipocyte liver kinase b1 suppresses beige adipocyte renaissance through class IIa histone deacetylase 4," Diabetes, vol. 66, no. 12, pp. 2952-2963, 2017.

[56] L. Chen, Q. Chen, P. Rong, H. Y. Wang, and S. Chen, "The energy sensing LKB1-AMPK $\alpha 1$ pathway regulates IGF1 secretion and consequent activation of the IGF1R-PKB pathway in primary hepatocytes," The FEBS Journal, vol. 284, no. 13, pp. 2096-2109, 2017.

[57] L. Pretorius, G. J. A. Thomson, R. C. M. Adams, T. A. Nell, W. A. Laubscher, and E. Pretorius, "Platelet activity and hypercoagulation in type 2 diabetes," Cardiovascular Diabetology, vol. 17, no. 1, p. 141, 2018.

[58] X. Huang, G. Liu, J. Guo, and Z. Su, “The PI3K/AKT pathway in obesity and type 2 diabetes," International Journal of Biological Sciences, vol. 14, no. 11, pp. 1483-1496, 2018.

[59] P. Abeyrathna and Y. Su, "The critical role of Akt in cardiovascular function," Vascular Pharmacology, vol. 74, pp. 38-48, 2015.

[60] F. J. Gonzalez, C. Xie, and C. Jiang, "The role of hypoxiainducible factors in metabolic diseases," Nature Reviews Endocrinology, vol. 15, no. 1, pp. 21-32, 2019.

[61] J. C. Jun, R. Devera, D. Unnikrishnan et al., "Adipose HIF-1 $\alpha$ causes obesity by suppressing brown adipose tissue thermogenesis," Journal of Molecular Medicine, vol. 95, no. 3, pp. 287-297, 2017.
[62] J. Hodge, T. Kawabata, S. Krishnaswami et al., "The mechanism of action of tofacitinib - an oral Janus kinase inhibitor for the treatment of rheumatoid arthritis," Clinical and Experimental Rheumatology, vol. 34, no. 2, pp. 318-328, 2016.

[63] E. N. Gurzov, W. J. Stanley, E. G. Pappas, H. E. Thomas, and D. J. Gough, "The JAK/STAT pathway in obesity and diabetes," The FEBS Journal, vol. 283, no. 16, pp. 30023015, 2016.

[64] E. Giovannucci, D. M. Harlan, M. C. Archer et al., "Diabetes and cancer: a consensus Report," CA: a Cancer Journal for Clinicians, vol. 60, no. 4, pp. 207-221, 2010.

[65] M. Jalving, J. A. Gietema, J. D. Lefrandt et al., "Metformin: taking away the candy for cancer?," European Journal of Cancer, vol. 46, no. 13, pp. 2369-2380, 2010.

[66] T. Kitamura, "The role of FOXO1 in $\beta$-cell failure and type 2 diabetes mellitus," Nature Reviews Endocrinology, vol. 9, no. 10, pp. 615-623, 2013.

[67] R. Yosef, N. Pilpel, N. Papismadov et al., "p21 maintains senescent cell viability under persistent DNA damage response by restraining JNK and caspase signaling," The EMBO Journal, vol. 36, no. 15, pp. 2280-2295, 2017.

[68] J. Taneera, J. Fadista, E. Ahlqvist et al., "Expression profiling of cell cycle genes in human pancreatic islets with and without type 2 diabetes," Molecular and Cellular Endocrinology, vol. 375, no. 1-2, pp. 35-42, 2013.

[69] R. Roskoski Jr., "RAF protein-serine/threonine kinases: structure and regulation," Biochemical and Biophysical Research Communications, vol. 399, no. 3, pp. 313-317, 2010.

[70] A. E. Butler, J. Janson, S. Bonner-Weir, R. Ritzel, R. A. Rizza, and P. C. Butler, "Cell deficit and Increased-Cell Apoptosis in humans with type 2 Diabetes," Diabetes, vol. 52, no. 1, pp. 102-110, 2003.

[71] P. Marchetti, S. Del Prato, R. Lupi, and S. Del Guerra, "The pancreatic beta-cell in human type 2 diabetes," Nutrition, Metabolism and Cardiovascular Diseases, vol. 16, pp. S3-S6, 2006.

[72] J. L. Beith, E. U. Alejandro, and J. D. Johnson, "Insulin stimulates primary $\beta$-cell proliferation via Raf-1 kinase," Endocrinology, vol. 149, no. 5, pp. 2251-2260, 2008.

[73] R. A. Tumor-linked, "HER2 expression: association with obesity and lipid-related microenvironment," Hormone Molecular Biology and Clinical Investigation, vol. 32, no. 3, 2017.

[74] P. Ferroni, S. Riondino, O. Buonomo, R. Palmirotta, F. Guadagni, and M. Roselli, "Type 2 diabetes and breast cancer: the interplay between impaired glucose metabolism and oxidant stress," Oxidative Medicine and Cellular Longevity, vol. 2015, Article ID 183928, 10 pages, 2015.

[75] A. A. Memon, L. Bennet, B. Zöller et al., "Circulating human epidermal growth factor receptor 2 (HER2) is associated with hyperglycaemia and insulin resistance," Journal of Diabetes, vol. 7, no. 3, pp. 369-377, 2015.

[76] J. E. Mindur and F. K. Swirski, "Growth factors as immunotherapeutic targets in cardiovascular Disease," Arteriosclerosis, Thrombosis, and Vascular Biology, vol. 39, no. 7, pp. 12751287, 2019.

[77] M. El-Daly, V. K. P. Venu, M. Saifeddine et al., "Hyperglycaemic impairment of PAR2-mediated vasodilation: prevention by inhibition of aortic endothelial sodium-glucose-co-Transporter-2 and minimizing oxidative stress," Vascular Pharmacology, vol. 109, pp. 56-71, 2018. 
[78] Z. Li, Y. Li, J. M. Overstreet et al., "Inhibition of epidermal growth factor receptor activation is associated with improved diabetic nephropathy and insulin resistance in type 2 diabetes," Diabetes, vol. 67, no. 9, pp. 1847-1857, 2018.

[79] Y. Xu, S. Xie, and Y. Ma, "Low expression of microRNA-15b promotes the proliferation of retinal capillary endothelial cells and pericytes by up-regulating VEGFA in diabetic rats," European Review for Medical and Pharmacological Sciences, vol. 23, no. 14, pp. 6018-6025, 2019. 